\title{
DETERMINAÇÃO HISTOPATOLÓGICA DA PRESENÇA DO Helicobacter pylori EM CÂNCER GÁSTRICO
}

\author{
Histopathological determination of Helicobacter pylori in gastric cancer
}

\author{
Claudio BRESCIANI, Ibrahim LATIF, Roger Beltrati COSER, Osmar YAGI, Claudio Roberto DEUTSCH, \\ Donato MUCERINO, Bruno ZILBERSTEIN, Ivan CECCONELLO
}

Trabalho realizado na Faculdade de Medicina da Universidade de São Paulo, Departamento de Gastroenterologia, , São Paulo, SP, Brasil.

DESCRITORES - Neoplasias gástricas. Helicobacter pylori.
RESUMO - Racional - A causa do câncer gástrico (CG) é controversa e tem vários fatores envolvidos no seu processo de carcinogênese, incluindo o Helicobacter pylori ( $\mathrm{Hp}$ ) O papel da infecção pelo Hp no CG permanece incerto, com vários estudos controversos. Objetivo - Correlacionar a presença da infecção pelo Hp com câncer gástrico, através de exame anatomopatológico convencional do estômago ressecado. Método - Noventa e um pacientes tratados por ressecção cirúrgica foram revistos. O exame anatomopatológico foi feito em todos os pacientes para determinar a presença de infecção por Hp, metaplasia intestinal (MI) e confirmação do tipo histológico por hematoxilina-eosina. A análise estatística foi realizada através do qui-quadrado e testes de log-rank. Resultados - MI foi observada em 81 tumores (89\%). Em geral, a presença de infecção pelo Hp foi observada em 46 casos (50,5\%). Não houve associação entre idade e Hp. Nos grupos de pacientes com CG avançado e precoce, a infecção pelo $\mathrm{Hp}$ estava presente em $47,7 \%$ e $54 \%$ dos tumores. A infecção pelo Hp ocorreu em 40 tumores (49\%) no grupo de pacientes com MI. Nos com tumores sem MI, Hp estava presente em cinco (50\%). Tumores proximais tiveram mais infecção por Hp, quando comparados aos tumores distais. Conclusões - A taxa de infecção não teve associação significativa com o tipo histológico, sexo, MI ou estágio de desenvolvimento tumoral. Esses resultados podem indicar que a participação da infecção pelo Hp durante o desenvolvimento do CG não pode ser descartada; no entanto, provavelmente não é essencial em todas as fases e o mecanismo do CG pode ser distinto da gastrite crônica e MI.Finalmente, é possível que a associação proposta é mera coincidência e que não há nenhuma influência real das bactérias no processo de carcinogênese.

\section{Correspondência: \\ Ivan Cecconello \\ e-mail:cecconello@terra.com.br \\ Fonte de financiamento: não há Conflito de interesses: não há}

Recebido para publicação: 16/09/2010 Aceito para publicação: 21/12/2010

HEADINGS - Stomach Neoplasms. Helicobacter pylori.
ABSTRACT - Introduction - Etiology of gastric cancer (GC) remains controversial and several factors have implicated in its carcinogenesis process, including Helicobacter pylori (Hp) infection. Hp infection's role on GC remains uncertain, with several conflicting studies. Method - Ninety-one patients with diagnosis of adenocarcinoma of the stomach treated by surgical resection were reviewed. Pathological examination was repeated in all patients to determine the presence of $\mathrm{Hp}$ infection, intestinal metaplasia (IM) and confirmation of the hystologic type by conventional haematoxylin-eosin staining. Statistical analysis was performed using Chi-quadrate and log-rank tests. Results - IM was observed in 81 tumours (89\%). Overall, the presence of $\mathrm{Hp}$ infection was observed in 46 tumours (50.5\%). There was no association between age and $\mathrm{Hp}$ status. In the group of patients with early and advanced GC, Hp infection was present in $47.7 \%$ and $54 \%$ of tumours. Hp infection was present in 40 tumours (49\%) in the group of patients with IM. In patients with tumours without IM Hp was present in five $(50 \%)$ tumours. Proximal tumours had more Hp infection when compared to distal tumours. Conclusions: The infection rate had no significant association with histologic type, IM, gender or stage. These results may indicate that participation of $\mathrm{Hp}$ infection during GC development cannot be ruled out; however, it is probably not essential during all stages of GC development and the mechanism may be distinct of the chronic gastritis and IM progression. Finally, it is possible that the proposed association is merely coincidental and that there is no actual influence of the bacteria in the carcinogenesis process. 


\section{INTRODUÇÃO}

Câncer gástrico (CG) continua sendo uma das neoplasias mais importantes a nível mundial, com significativa incidência e mortalidade ${ }^{27,33,35,39}$. Sua causa permanece controversa e vários fatores têm sido implicados no seu processo de carcinogênese. Entre esses fatores, os eventos genéticos, ambientais, e características da dieta parecem ser relevantes ${ }^{3,19,33,35}$.

Helicobacter pylori ( $\mathrm{Hp}$ ) também tem sido implicado na carcinogênese do câncer gástrico. Esta associação tem sido sugerida pela observação de taxas mais elevadas de infecção pelo $\mathrm{Hp}$ em pacientes com câncer gástrico, quando comparados aos controles normais em países ocidentais ${ }^{6,7,25}$. Por outro lado, em países com alta incidência da neoplasia, a prevalência da infecção pelo Hp parece ser ainda maior, ocorrendo em idades precoces da população em geral ${ }^{13,25}$.

O papel da infecção pelo $\mathrm{Hp}$ no desenvolvimento do câncer gástrico pode estar associado com a produção de citocinas específicas e enzimas levando à gastrite crônica atrófica e metaplasia intestinal ${ }^{3,19,35}$. Contudo, esta associação ainda é controversa, alguns estudos relatam a associação de $\mathrm{Hp}$ infecção de gastrite crônica, mas não para câncer gástrico ${ }^{13}$. Além disso, alguns países com baixa incidência de câncer gástrico podem apresentar elevada prevalência de infecção pelo $\mathrm{Hp}^{8}$.

Por estas razões, em um país com elevada prevalência da infecção pelo $\mathrm{Hp}$ entre a população em geral como no Brasil, seria de se esperar encontrar altas taxas de infecção pelo $\mathrm{Hp}$ em pacientes com CG. Também, seria de se esperar associação entre infecção pelo Hp, metaplasia intestinal (MI) ou tipo intestinal CG.

O objetivo deste estudo foi verificar a presença de infecção pelo Hp em pacientes com câncer gástrico, através de exame anatomopatológico convencional do estômago ressecado.

\section{MÉTODOS}

Foram revisados noventa e um pacientes com diagnóstico desta neoplasia e tratados por ressecção cirúrgica entre 2000 e 2002. Os dados coletados incluíram idade ao diagnóstico, sexo, localização do tumor, tipo histológico (de acordo com a classificação de Lauren), relatório anatomopatológico completo e estágio de evolução da doença de acordo com a UICC1998.

Quarenta mulheres (44\%) e 51 (56\%) homens foram elegíveis para o estudo. A idade média foi de 61 variando de 19 a 87 anos. Três pacientes (3,3\%) tiveram seus tumores localizados no terço proximal $(U)$, $33(36,3 \%)$, no terço médio $(\mathrm{M})$ e $42(46,1 \%)$ no terço distal $(\mathrm{L})$ do estômago. Treze pacientes tinham tumores localizados em mais de uma região, sendo oito $(8,8 \%)$ nos terços distal e médio (LM), dois $(2,2 \%)$ no terço proximal e médio (UM) e três tumores (3,3\%) no órgão inteiro (LMU). A fim de realizar análises estatísticas sobre a localização, os tumores de localização proximal e média foram agregados e nomeados "tumores proximais".

O exame patológico foi revisto por um patologista experiente em todos os pacientes, a fim de determinar a presença de infecção pelo $\mathrm{Hp}$, metaplasia intestinal (MI) e a confirmação do tipo histológico (classificação de Lauren) por hematoxilina-eosina. A presença de infecção pelo $\mathrm{Hp}$ foi determinada por visualização direta da bactéria na mucosa normal adjacente de câncer gástrico.

Correlação entre infecção e HP, características epidemiológicas, clínicas e patológicas foram realizadas. A análise estatística foi feira através do quiquadrado e testes de log-rank.

\section{RESULTADOS}

No geral, 61 pacientes (68\%) tinham o tipo intestinal de adenocarcinoma de acordo com a classificação de Laurén, enquanto 30 (32\%) tiveram o tipo difuso. Sessenta e sete pacientes (74\%) tinham CG precoce, enquanto 24 (26\%) avançado. No grupo de pacientes com CG precoce, 21 (31\%) tiveram o tipo difuso e 46 (69\%) tipo intestinal. No grupo de pacientes com CG avançado, nove (30\%) apresentaram tipo difuso e 15 (70\%) intestinal (Tabela 1$)$.

TABELA 1 - População estudada $(n=91)$

\begin{tabular}{|c|c|}
\hline \multicolumn{2}{|c|}{ População estudada } \\
\hline \multicolumn{2}{|l|}{ Sexo } \\
\hline Feminino & 40 (44\%) \\
\hline Masculino & $51(56 \%)$ \\
\hline \multicolumn{2}{|l|}{ Localização tumoral } \\
\hline Superior & $3(3,3 \%)$ \\
\hline Média & $33(36,3 \%)$ \\
\hline Inferior & $42(46,1 \%)$ \\
\hline Média/inferior & $8(8,8 \%)$ \\
\hline Superior/média & $2(2,2 \%)$ \\
\hline Todo o estômago & $3(3,3 \%)$ \\
\hline \multicolumn{2}{|l|}{ Tipo histológico } \\
\hline Intestinal & 61 (68\%) \\
\hline Difuso & $30(32 \%)$ \\
\hline \multicolumn{2}{|l|}{ Fase do CG } \\
\hline Precoce & 67 (74\%) \\
\hline Avançado & 24 (26\%) \\
\hline
\end{tabular}

Metaplasia intestinal (MI) associada foi observada em 81 tumores (89\%). No grupo de pacientes com tipo intestinal, ela esteve presente em 54 tumores (88\%). No grupo de pacientes com GC difusa, MI foi observada em 27 tumores (90\%). No grupo de pacientes com câncer 
gástrico precoce, 63 (94\%) tumores tinham metaplasia intestinal, enquanto que no grupo avançado, 21 (87\%) tumores tinham IM. Não houve correlação significativa entre a presença de MI, estágio avançado/precoce ou tipo histológico $(p>0,05)$ (Tabela 2).

TABELA 2 - Metaplasia intestinal de acordo com as características do tumor

\begin{tabular}{|c|c|c|c|}
\hline Metaplasia intestinal & MI - & $\mathrm{MI}+$ & $\mathrm{p}$ \\
\hline $\mathrm{N}$ & 10 (11\%) & 81 (89\%) & \\
\hline \multicolumn{4}{|l|}{ Tipo histológico } \\
\hline Intestinal & 7 (12\%) & 54 (88\%) & \\
\hline Difuso & $3(10 \%)$ & 27 (90\%) & ns \\
\hline \multicolumn{4}{|l|}{ Fase do CG } \\
\hline Precoce & $4(6 \%)$ & 63 (94\%) & \\
\hline Avançado & $3(13 \%)$ & 21 (87\%) & ns \\
\hline
\end{tabular}

Em geral, a presença da infecção pelo $\mathrm{Hp}$ foi observada em 46 tumores (50,5\%). Frequências semelhantes foram observadas em homens (45\%) e mulheres (55\%). Não houve associação entre idade e estado de $\mathrm{Hp}$. Em pacientes com CG do tipo difuso, $\mathrm{Hp}$ estava presente em 14 tumores (47\%), enquanto nos pacientes com tipo intestinal, presente em 31 tumores (51\%). Além disso, no grupo de pacientes com CG precoce, Hp era positivo em 32 tumores $(47,7 \%)$, enquanto nos avançado em 13 pacientes (54\%). Estas diferenças não apresentaram significância estatística $(p>0,05)$.

A infecção pelo $\mathrm{Hp}$ estava presente em 40 tumores (49\%) com MI. Em pacientes com tumores sem MI, Hp estava presente em cinco (50\%). No subgrupo com CG do tipo intestinal e com o MI a presença de $\mathrm{Hp}$ foi detectada em 27 tumores (50\%). Estas diferenças não apresentaram significância estatística $(p>0,05)$. Curiosamente, em pacientes com CG localizado no terço distal do estômago, a presença de infecção pelo $\mathrm{Hp}$ foi observada em 14 tumores (37\%). Nos pacientes com CG localizado proximalmente, ela estava presente em 28 casos (56\%). Tumores proximais tiveram maior frequência de infecção $\mathrm{Hp}$ quando comparados aos tumores distais $(p=0,03)$ (Tabela 3).

DISCUSSÃO

No início de 1900, a presença de Hp na superfície da mucosa gástrica foi descrita pela primeira vez, coincidentemente em uma amostra de câncer gástrico ${ }^{16}$. No entanto, somente no início dos anos 80 foi proposta relação entre a infecção pelo $\mathrm{Hp}$ e doenças do estômago $0^{7,11,18}$.

Durante a infecção inicial pelo $\mathrm{Hp}$, infiltrado
TABELA 3 - Determinação Helicobacter pylori por exame antomopatológico (HE)

\begin{tabular}{|l|c|c|c|}
\hline Infecção pelo Hp & $\mathrm{Hp}-$ & $\mathrm{Hp}+$ & $\mathrm{p}$ \\
\hline $\mathrm{N}$ & $45(49,5 \%)$ & $46(50,5 \%)$ & \\
\hline $\begin{array}{l}\text { Sexo } \\
\text { Feminino }\end{array}$ & $18(45 \%)$ & $22(55 \%)$ & \\
\hline $\begin{array}{l}\text { Masculino } \\
\text { Localização tumoral }\end{array}$ & $28(55 \%)$ & $23(45 \%)$ & $\mathrm{ns}$ \\
\hline $\begin{array}{l}\text { Proximal (superior, superior/ } \\
\text { médio e médio) }\end{array}$ & $22(44 \%)$ & $28(56 \%)$ & \\
\hline $\begin{array}{l}\text { Distal (inferior e inferior/médio) } \\
\text { Todo o estômago }\end{array}$ & $23(63 \%)$ & $14(37 \%)$ & \\
\hline Tipo histológico & - & $3(100 \%)$ & $\mathrm{p}=0,03$ \\
\hline $\begin{array}{l}\text { Intestinal } \\
\text { Difuso }\end{array}$ & $31(51 \%)$ & $30(49 \%)$ & \\
\hline $\begin{array}{l}\text { Fase do CG } \\
\text { Precoce }\end{array}$ & $14(47 \%)$ & $16(53 \%)$ & $\mathrm{ns}$ \\
\hline $\begin{array}{l}\text { Avançado } \\
\text { Metaplasia intestinal }\end{array}$ & $32(47,7 \%)$ & $35(52,3 \%)$ & \\
\hline $\begin{array}{l}\text { Presente } \\
\text { Ausente }\end{array}$ & $13(54 \%)$ & $11(46 \%)$ & $\mathrm{ns}$ \\
\hline
\end{tabular}

inflamatório polimórfico neutrofílico é observado na superfície gástrica na fase aguda. Posteriormente, há diminuição na produção de muco, resultando em diminuição na espessura da mucosa. A infecção crônica é caracterizada por aumento na contagem de linfócitos e plasmócitos na lâmina própria19,29,33,35.

Proporção significativa de pacientes com infecção pelo $\mathrm{Hp}$ permanecem assintomáticos apresentando gastrite crônica ativa ${ }^{21,28,33,35}$. Alguns podem desenvolver úlceras. Uma minoria com infecção pelo $\mathrm{Hp}$ desenvolve gastrite crônica atrófica e metaplasia intestinal ${ }^{17,32}$. A progressão para a doença podem ser influenciados por fatores dietéticos, como vitamina $C^{3,23,24}$, sal e ingestão de nitrato 9,23,24. O mecanismo proposto para a progressão para gastrite atrófica e metaplasia intestinal crônica pode estar associado com a produção de citocinas e enzimas com ação direta ou indireta sobre a mucosa gástrica ${ }^{33}$. Além disso, o processo inflamatório na parede gástrica pode levar à maior produção de citocinas, tais como IL-6, IL-8, TNF-alfa e peróxidos contribuindo para o dano da mucosa ${ }^{10,15}$. Atividade da urease do Hp, por exemplo, pode resultar na produção de amônia, que por sua vez, diminui a integridade da mucosa e eleva o pH da secreção gástrica favorecendo a proliferação bacteriana ${ }^{19,33,35}$.

O mecanismo de ação proposto pelo $\mathrm{Hp}$ na gênese do CG é através do desenvolvimento de gastrite crônica e metaplasia intestinal6,31,36. Estas situações podem ser consideradas nas condições pré-malignas, especificamente para o tipo de câncer gástrico intestinal e, portanto, infecção pelo $\mathrm{Hp}$ pode ser também considerado por alguns como pré-maligna ${ }^{6,12,19,36}$.

Nesse cenário, seria de grande interesse determinar a frequência da infecção pelo $\mathrm{Hp}$ em CG. Seria de se esperar encontrar taxas mais elevadas de $\mathrm{Hp}$ em pacientes com CG, quando comparados a controles normais na população em geral. Além disso, se o mecanismo de 
ação da infecção pelo Hp em CG é considerado, seria esperado encontrar ainda maiores taxas de infecção pelo Hp em câncer intestinal associada com metaplasia intestinal. A confirmação de tais proposições favoreceria a participação do $\mathrm{Hp}$ na carcinogênese gástrica e força a importância da erradicação dele em pacientes assintomáticos, incluindo a possibilidade de medidas de prevenção populacional, tais como vacinação em massa de áreas de alto risco para reduzir a incidência de CG6,28,31,33.

Determinação da infecção pelo $\mathrm{Hp}$ em alguns países, como Holanda e Reino Unido, apresentaram índices maiores em pacientes com CG (61-69\%) quando comparada a indivíduos normais (31-47\%) ${ }^{6}$. No entanto, em outras regiões, como na Itália, estas diferenças não foram observadas ${ }^{26,37}$. Além disso, regiões com alta incidência de CG parece ter altas prevalências de infecção pelo $\mathrm{Hp}$, como o Japão, onde aproximadamente $75 \%$ da população em geral tem evidência sorológica de infecção, onde a incidência CG é muito elevada em comparação com outros países e do mundo ocidental ${ }^{1,6}$. Nesse país, considera-se que há risco aumentado em três para o desenvolvimento da CG em indivíduos com infecção pelo $\mathrm{Hp}$ quando comparados aos indivíduos livres $\mathrm{Hp}$, desde que a grande maioria das infecções parece ocorrer precocemente durante a vida ${ }^{4,6}$. Por outro lado, na África, onde há alta prevalência de infecção pelo $\mathrm{Hp}$ na população em geral, há incidência muito baixa de CG8,31. Além disso, a alta frequência de tipo intestinal e distal em idosos pode facilitar a proliferação bacteriana, sendo a infecção $\mathrm{Hp}$ meramente circunstancial ${ }^{22}$. Finalmente, o baixo estado dos países em desenvolvimento pode estar associado com altas taxas de doenças infecciosas (incluindo a infecção pelo Hp). Especificamente no Brasil, onde a prevalência de doenças infecciosas e GC são altos, a associação entre elas pode ser mera coincidência.

Há algumas outras evidências que não favorecem correlação entre a infecção por $\mathrm{Hp}$ e carcinogênese no CG. Em primeiro lugar, a associação entre a infecção pelo $\mathrm{Hp}$ e gastrite crônica está bem estabelecida, mas a associação entre a infecção pelo Hp e CG não é amplamente aceita ${ }^{31,38}$. Alguns autores acreditam que a CG surge "de novo" e não através de gastrite crônica e metaplasia intestinal ${ }^{13,38}$. Além disso, há associação significativa entre o CG e sexo (homens são mais frequentemente afetados). Portanto, seria de se esperar encontrar a mesma associação entre sexo e infecção pelo Hp. No entanto, a prevalência da infecção dele mostra a distribuição igualitária entre homens e mulheres controles, incluindo os resultados observados no presente estudo em pacientes com $\mathrm{CG}^{38}$.

Neste estudo, a determinação da infecção pelo $\mathrm{Hp}$ em 91 amostras de CG foi feita por exame anatomopatológico convencional, utilizando hematoxilina-eosina. Este método é muito caro, minucioso e a presença de um patologista experiente é crucial. Por outro lado dá informação inequívoca sobre a presença de infecção pelo Hp. Além disso, ele pode ser útil em casos de CG, onde ressecção cirúrgica, foi realizada, mas questionável em controles normais em biópsias endoscópicas por vezes escassas e insuficientes para a determinação exata da infecção pelo Hp.

A prevalência da infecção pelo $\mathrm{Hp}$ neste estudo foi surpreendentemente baixa, de 50,5\%. Um estudo anterior realizado em nosso país, com 18 pacientes com CG, mostrou prevalência de infecção de mais de $80 \%$, muito superior a estes resultados ${ }^{25}$. Além disso, a taxa de infecção no presente estudo é consideravelmente menor do que a prevalência da infecção em controles normais no Brasil, determinado por estudos sorológicos ${ }^{20,30}$. Nosso país é considerado como apresentando alta prevalência de infecção na população geral, atingindo taxas de infecção de até $80 \%{ }^{20,30}$. A infecção pelo Hp em pacientes com CG neste estudo ocorreram em taxas similares entre ambos os sexos, de acordo com observações anteriores em controles normais ${ }^{1,30,38}$.

Curiosamente, houve correlação significativa entre a infecção pelo $\mathrm{Hp}$ e localização do tumor no CG. No entanto, a baixa incidência de tumores proximais pode ser responsável por esta diferença, já que por razões estatísticas, tumores localizados no terço proximal e médio tiveram que ser agregados. Surpreendentemente, a taxa de infecção não teve associação significativa com o tipo histológico, sexo ou estágio (inicial vs avançado), quando deveria serem esperadas taxas mais altas de infecção do tipo intestinal. Surpreendentemente, não houve associação entre a infecção pelo Hp e MI, uma das evidências propostas para correlação da infecção pelo Hp e CG.

\section{CONCLUSÃO}

Os resultados da infecção pelo $\mathrm{Hp}$ em pacientes com CG indicam que ela ocorre aproximadamente em $50 \%$ dos pacientes pelo estudo histopatológico do espécime ressecado. Participação dele durante o desenvolvimento do CG não pode ser descartada, porém provavelmente não é essencial durante todas as fases do desenvolvimento da doença. Além disso, se houver a participação da infecção pelo $\mathrm{Hp}$ no desenvolvimento do câncer gástrico, o mecanismo pode ser distinto do da gastrite crônica e a progressão MI. Finalmente, é possível que a associação entre a infecção pelo Hp e CG é mera coincidência e que não há nenhuma influência real das bactérias no processo de carcinogênese.

\section{REFERÊNCIAS}

1. An international association between Helicobacter pylori infection and gastric cancer. The EUROGAST Study Group. Lancet 1993;341(8857):1359-62.

2. Helicobacter pylori in patients with intestinal metaplasia and in controls: a serological and biopsy study in four UK centres. UK Sub-Group of the ECP-EURONUT-Intestinal Metaplasia Study Group. Eur J Cancer Prev 1995;4(2):175-80. 
3. Asaka $M$, Takeda $H$, Sugiyama $T$, Kato $M$. What role does Helicobacter pylori play in gastric cancer? Gastroenterology 1997;113(6 Suppl):S56-60.

4. Barreto R, Maruyama M, Kato Y, Aizu K. Gastric cancer and Helicobacter pylori infection: a seroprevalence study in Cancer Institute Hospital Japan. In: 1st International Gastric Cancer Congress.; 1995; Kyoto, Japan.

5. Craanen ME, Blok P, Dekker W, Tytgat GN. Helicobacter pylori and early gastric cancer. Gut 1994;35(10):1372-4.

6. Endo S, Ohkusa T, Saito Y, Fujiki K, Okayasu I, Sato C. Detection of Helicobacter pylori infection in early gastric cancer. A comparison between intestinal and diffuse-type gastric adenocarcinomas. Cancer 1995;75(9):2203-8.

7. Fox JG, Correa P, Taylor NS, Zavala D, Fontham E, Janney F, Rodriguez E, Hunter F, Diavolitsis S. Campylobacter pylori-associated gastritis and immune response in a population at increased risk of gastric carcinoma. Am J Gastroenterol 1989;84(7):775-81.

8. Holcombe C. Helicobacter pylori: the African enigma. Gut 1992;33(4):429-31.

9. Hu JF, Zhang SF, Jia EM, Wang QQ Liu SD, Liu YY, Wu YP, Cheng YT. Diet and cancer of the stomach: a case-control study in China. Int J Cancer 1988;41(3):331-5.

10.Innocenti $M$, Thoreson AC, Ferrero RL, Strömberg E, Bölin I, Eriksson L, Svennerholm AM, Quiding-Järbrink M. Helicobacter pylori-induced activation of human endothelial cells. Infect Immun 2002;70(8):4581-90.

11. Jaskiewicz K, Louwrens HD, Woodroof CW, van Wyk MJ, Price SK. The association of Campylobacter pylori with mucosal pathological changes in a population at risk for gastric cancer. $\mathrm{S}$ Afr Med J 1989;75(9):417-9.

12. Kato S, Onda M, Matsukura N, Tokunaga A, Matsuda N, Yamashita K, Shields PG. Helicobacter pylori infection and genetic polymorphisms for cancer-related genes in gastric carcinogenesis. Biomed Pharmacother 1997;51(4):145-9.

13. Kato T, Saito Y, Matsumura S, Akiyama N. Helicobacter pylori infection in early gastric cancer. In: 1st International Gastric Cancer Congress; 1995; Kyoto, Japan.

14. Kelley JR, Duggan JM. Gastric cancer epidemiology and risk factors. J Clin Epidemiol 2003;56(1):1-9.

15. Klausz G, Tiszai A, Tiszlavicz L, Gyulai Z, Lénárt Z, Lonovics J, Mándi Y. Local and peripheral cytokine response and CagA status of Helicobacter pylori-positive patients with duodenal ulcer. Eur Cytokine Netw 2003;14(3):143-8.

16. Krienitz W. Ueber das Auftreten von spirochaten verschiedener form im mageninhalt bei carcinoma ventriculi. Dtsch Med Wochenschr 1906;332872.

17. Kuipers EJ, Uyterlinde AM, Peña AS, Roosendaal R, Pals G, Nelis GF, Festen HP, Meuwissen SG. Long-term sequelae of Helicobacter pylori gastritis. Lancet 1995;345(8964):1525-8.

18. Marshall BJ, Warren JR. Unidentified curved bacilli in the stomach of patients with gastritis and peptic ulceration. Lancet 1984;1(8390):1311-5.

19. Matsukura N, Onda M, Tokunaga A. Mucosal IgA antibody against Helicobacter pylori in chronic gastritis and intestinal metaplasia in resected stomach for gastric cancer. In: $1^{\text {st }}$ International Gastric Cancer Congress; 1995; Kyoto, Japan.

20. Mattar R, Silva F, Birbojm I, Zilberstein B. Helicobacter pylori infection: a risk factor for gastric cancer? Serological study. In: Fifth International Gastric Cancer Congress; 2003; Rome, Italy.

21. McFarlane GA, Munro A. Helicobacter pylori and gastric cancer. $\mathrm{Br}$ J Surg 1997;84(9):1190-9.

22. Megraud F. Epidemiology of Helicopbacter pylori infection. In: Rathbone Be, RV, eds. (ed) Helicobacter pylori and Gastroduodenal
Disease. 2nd ed. Blachwell Scientific Piblications; 1992.

23. Mirvish SS. The etiology of gastric cancer. Intragastric nitrosamide formation and other theories. J Natl Cancer Inst 1983;71(3):62947.

24. Mirvish SS. Effects of vitamins $\mathrm{C}$ and $\mathrm{E}$ on $\mathrm{N}$-nitroso compound formation, carcinogenesis, and cancer. Cancer 1986;58(8 Suppl):1842-50.

25. Nogueira AM, Ribeiro GM, Rodrigues MA, Queiroz DM, Mendes EN, Rocha GA, Barbosa AJ. Prevalence of Helicobacter pylori in Brazilian patients with gastric carcinoma. Am J Clin Pathol 1993;100(3):236-9.

26. Palli D, Decarli A, Cipriani F, Sitas F, Forman D, Amadori D, Avellini C, Giacosa A, Manca P, Russo A. Helicobacter pylori antibodies in areas of Italy at varying gastric cancer risk. Cancer Epidemiol Biomarkers Prev 1993;2(1):37-40.

27. Parkin DM, Muir CS. Cancer Incidence in Five Continents. Comparability and quality of data. IARC Sci Publ 1992(120):45173.

28. Parsonnet J, Harris RA, Hack HM, Owens DK. Modelling costeffectiveness of Helicobacter pylori screening to prevent gastric cancer: a mandate for clinical trials. Lancet 1996;348(9021):150-4.

29. Price A. The Sidney system: histological division. J Gastroenterol Hepatol 1991;6209-22.

30. Rocha GA, Queiroz DM, Mendes EN, Oliveira AM, Moura SB, Barbosa MT, Mendes CC, Lima Júnior GF, Oliveira CA. Indirect immunofluorescence determination of the frequency of anti- $\mathrm{H}$. pylori antibodies in Brazilian blood donors. Braz J Med Biol Res 1992;25(7):683-9.

31. Rugge M, Cassaro M, Leandro G, Baffa R, Avellini C, Bufo P, Stracca V, Battaglia G, Fabiano A, Guerini A, Di Mario F. Helicobacter pylori in promotion of gastric carcinogenesis. Dig Dis Sci 1996;41(5):9505.

32. Sakaki N, Momma K, Egawa N, Yamada Y, Kan T, Ishiwata J. The influence of Helicobacter pylori infection on the progression of gastric mucosal atrophy and occurrence of gastric cancer. Eur J Gastroenterol Hepatol 1995;7 Suppl 1S59-62.

33. Scheiman JM, Cutler AF. Helicobacter pylori and gastric cancer. Am J Med 1999;106(2):222-6.

34. Solcia E, Fiocca R, Luinetti O, Villani L, Padovan L, Calistri D, Ranzani GN, Chiaravalli A, Capella C. Intestinal and diffuse gastric cancers arise in a different background of Helicobacter pylori gastritis through different gene involvement. Am J Surg Pathol 1996;20 Suppl 1S8-22.

35. Stolte M, Meining A. Helicobacter pylori and Gastric Cancer. Oncologist 1998;3(2):124-8.

36. Takeuchi K, Ohno Y, Tsuzuki Y, Ando T, Sekihara M, Hara T, Kuwano $\mathrm{H}$. Helicobacter pylori infection and early gastric cancer. J Clin Gastroenterol 2003;36(4):321-4.

37. Tomaselli G, Citarda F, Crespi M. Helicobacter pylori and gastric cancer. Clin Ter 1999;150(3):221-4.

38. Watanabe $\mathrm{Y}$, Kurata JH, Mizuno S, Mukai M, Inokuchi H, Miki K, Ozasa K, Kawai K. Helicobacter pylori infection and gastric cancer. A nested case-control study in a rural area of Japan. Dig Dis Sci 1997;42(7):1383-7.

39. Wingo PA, Tong T, Bolden S. Cancer statistics, 1995. CA Cancer J Clin 1995;45(1):8-30. 